\title{
Two layer dielectric-electrolyte micro-flow with pressure gradient
}

\author{
Georgy Ganchenko ${ }^{1, a}$, Abhishek Navarkar², Michael Zhukov ${ }^{3}$ and Sakir Amiroudine ${ }^{2}$ \\ ${ }^{1}$ Laboratory of Micro- and Nanoscale Electro- and Hydrodynamics for the Krasnodar Branch of Financial University, \\ 34 Luzana st., Krasnodar, 350051, Russia. \\ 2Université Bordeaux, I2M, UMR CNRS 5295, 16 Av. Pey-Berland, 33607 Pessac, France. \\ ${ }^{3}$ Department of computational mathematics and mathematical physics, Southern Federal University, 8a Melchakova \\ st., Rostov-on-Don, 344090, Russia.
}

\begin{abstract}
The present work considers stability of two-phase dielectric/electrolyte system, consisting of two immiscible liquids in microchannel. The system is set in motion by the constant external electric field, which causes the electroosmotic flow in the electrolyte, and by the pressure driving force. The investigation of its linear stability has shown that there are two types of instability in the system: short-wave and long-wave instability. The short-wave instability occurs for a stronger external field than the long-wave instability but the growth rate of the short-wave instability is much higher than that of the long-wave instability.
\end{abstract}

\section{Introduction}

Phenomena of micro- and nanoscale moving of conductive and non-conductive liquids with an interface between them requires a great deal of attention because it has numerous practical applications in biochemical processing: lab-on-cheap reactors, micromixers, DNA extraction, drug delivery and many others. The present work considers a two-layer micro/nanoflow of conductive (electrolyte) and nonconductive (dielectric) viscous liquids bounded by two solid walls in an external electric field and pressure gradient (see Fig. 1).

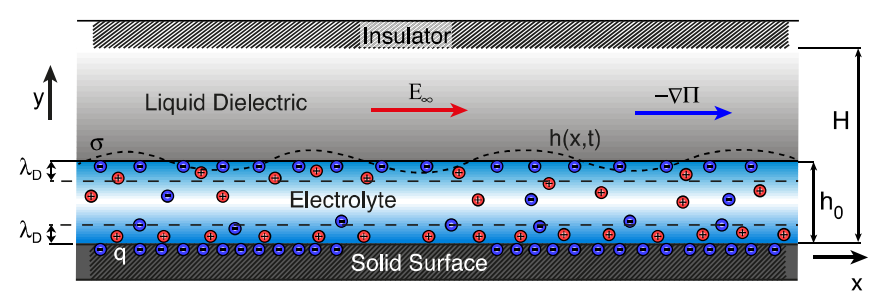

Figure 1. Electro-osmotic two-phase electrolyte-dielectric flow under an external tangential electric field and pressure gradient.

\footnotetext{
${ }^{\text {a }}$ Corresponding author ganchenko.ru@gmail.com
} 
The electrolyte-dielectric and the solid-electrolyte interfaces are assumed to be charged, while the solid-dielectric interface is an electrically insulated surface. The charge near the solid is immobile, while the surface charge on the electrolyte-dielectric interface is mobile [1] and references therein. Upon a tangential electric field, these charges produce a Coulomb force and cause a movement of the liquid; experimental evidence for an electroosmotic flow at the liquid-liquid interface and in the liquid bulk is given in [2]. In spite of almost zero Reynolds numbers (negligible inertia effects), the electrohydrodynamics of liquid flows at micro- and nanoscales includes complicated instabilities [3] and bifurcations [4], and even chaotic motion at micro- and nanoscales, which is named as microturbulence.

\section{Formulation of the problem}

The problem is described by the Nernst-Planck-Poisson-Stokes (NPPS) system in the liquidelectrolyte phase,

$$
\begin{gathered}
\frac{\partial c^{ \pm}}{\partial t}+\mathbf{U} \cdot \nabla c^{ \pm}= \pm \nabla \cdot\left(c^{ \pm} \nabla \Phi\right)+\nabla^{2} c^{ \pm} \\
v^{2} \nabla^{2} \Phi=c^{-}-c^{+} \\
\nabla P=\nabla^{2} \mathbf{U}-\frac{\kappa}{v^{2}} \nabla \Phi\left(c^{+}-c^{-}\right), \quad \nabla \cdot \mathbf{U}=0 .
\end{gathered}
$$

Here, $c^{ \pm}$are the molar concentrations of cations and anions; $\mathbf{U}$ is the fluid velocity vector; $\Phi$ is the electrical potential in the liquid phase; $P$ is the pressure.

The lower solid wall has a uniform surface charge, $q$, and provides the following boundary condition,

$$
y=0: \frac{\partial \Phi}{\partial y}=\frac{q}{v}
$$

The solid surface is assumed to be impermeable to both kinds of ions, and the velocity components obey no-slip BCs,

$$
\frac{\partial c^{ \pm}}{\partial y} \pm c^{ \pm} \frac{\partial \Phi}{\partial y}=0, \quad \mathbf{U}=0
$$

The BCs at the liquid-liquid interface, $y=h(x, t)$, are as follows,

(i) The surface is assumed to be impermeable to cations and anions:

$$
y=h(x, t): \frac{\partial c^{ \pm}}{\partial n} \pm c^{ \pm} \frac{\partial \Phi}{\partial n}=0 ;
$$

(ii) The potential is continuous while its gradient has a jump connected with a mobile surface charge

$$
\Phi=\phi, \frac{\partial \Phi}{\partial n}=\delta \frac{\partial \phi}{\partial n}+\frac{\sigma}{v}
$$

Here $\phi$ is the electrical potential in the dielectric phase.

(iii) The surface charge obeys the conservation law

$$
\frac{\partial \sigma}{\partial t}+\frac{\partial\left(U_{S} \sigma\right)}{\partial x}=0
$$

Here $U_{s}$ is the tangential component of the liquid-liquid interface velocity; exchanges between the surface charge and the bulk charge are neglected.

(iv) The stress-balance BCs at the interface $y=h(x, t))$ are as follows,

$$
(P-p)-\frac{W e}{R}+\left[\mathbf{n} \mathbf{T}^{f} \mathbf{n}\right]+\left[\mathbf{n} \mathbf{T}^{e} \mathbf{n}\right]=0, \quad\left[\mathbf{n} \mathbf{T}^{f} \mathbf{s}\right]+\left[\mathbf{n} \mathbf{T}^{e} \mathbf{s}\right]=0 .
$$

Here $p$ is the pressures in the dielectric, We is the Weber number, $\mathrm{R}$ is the radius of the curvature of the surface so that $W e / R$ is the capillary jump across the interface, $\mathbf{T}^{f}$ is the viscous, $\mathbf{T}^{e}$ is the Maxwell stress. Square brackets $[x]$ are the jump of value $x$ across the interface.

(v) The kinematic constraint at the interface, $V=\partial h / \partial t+U \partial h / \partial x$. 
The dielectric is described by the Laplace-Stokes system,

$$
\nabla^{2} \phi=0,-\nabla p+\mu \nabla^{2} \mathbf{u}=0, \nabla \cdot \mathbf{u}=0
$$

with the BCs on the upper boundary,

$$
y=H: \frac{\partial \phi}{\partial y}=0, \mathbf{u}=0
$$

The above problem is described by dimensionless control parameters: $E_{\infty}$ is the external electric filed, $\Pi$ is the external pressure field, $v$ is the Debye number $\delta$, is the ratio of the electric permittivities of the dielectric and electrolyte, $\mu$ is the ratio of the viscosities of the dielectric and electrolyte, $\kappa$ is a coefficient of coupling between the hydrodynamics and the electrostatics, $W e$ is the Weber number, $q$ is the dimensionless surface charge, $H$ is the dimensionless width of the channel. The surface charge at the interface is a parameter depending on $x$ and $t, \sigma=\sigma(x, t)$, but for one-dimensional solution one need to set a mean value of surface charge $\sigma_{0}$, so an additional parameter occurs in the system. The characteristic quantities and value of dimension parameters can be found in [1]. We set in our calculations $\kappa=0.2, q=-3, \sigma_{0}=1, v=0.1$, unless otherwise indicated.

\section{Basic steady-state solution}

The problem has 1D steady-state equilibrium solution with a plug-like velocity profile. Since the electrostatic and the hydrodynamic parts of the problem are decoupled in one-dimensional case and can be solved independently from each other, the results obtained in electrostatic part, are the same as in [1], where the dielectric liquid was replaced by the simpler case of air. The analysis of the equations one can found in [1].

Velocity profiles for different values of $\mu$ are shown in Fig. 2(a). As illustrated, the dielectric velocity decreases with increase of $\mu$. This is a natural behavior for the system with rather big values of viscosity of passive dielectric layer.

Electroosmotic flow has different directions, which depend on the direction of the external electric field, determined by the sign of $E_{\infty}$. If $E_{\infty}<0$, then the electroosmotic flow is parallel to the pressure driven flow, and vice versa, for $E_{\infty}>0$, the electroosmotic flow is anti-parallel, Fig 2(b)
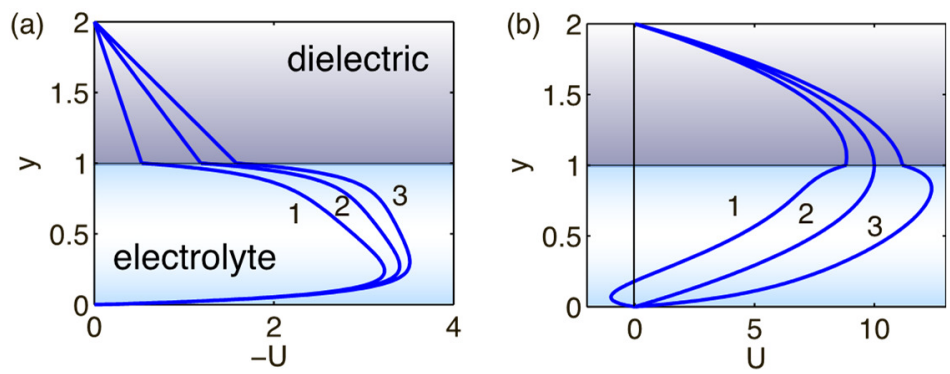

Figure 2. Velocity profile (a) for $\Pi=0, E_{\infty}=10$ and different values of $\mu 1: \mu=3.5,2: \mu=1,3: \mu=0.5$ and (b) for $\Pi=20, \mu=1$ and different values of $E_{\infty} 1: E_{\infty}=10,2: E_{\infty}=0,3: E_{\infty}=-10$.

\section{Numerical linear stability analysis}

Linear stability of the 1D steady-state equilibria in response to sinusoidal, with wave number $\alpha$, perturbations is considered. The stability investigation represents the solution to this problem in terms of a solution to an eigenvalue problem for a system of linear ordinary differential equations; the eigenvalue problem is solved numerically. The results show the coexistence of a long-wave, Fig. 3(b), and short-wave, Fig. 3(a), instabilities. The short-wave instability occurs for a stronger external field 
than the long-wave instability, but the growth rate of the short-wave instability is much higher than that of the long-wave instability
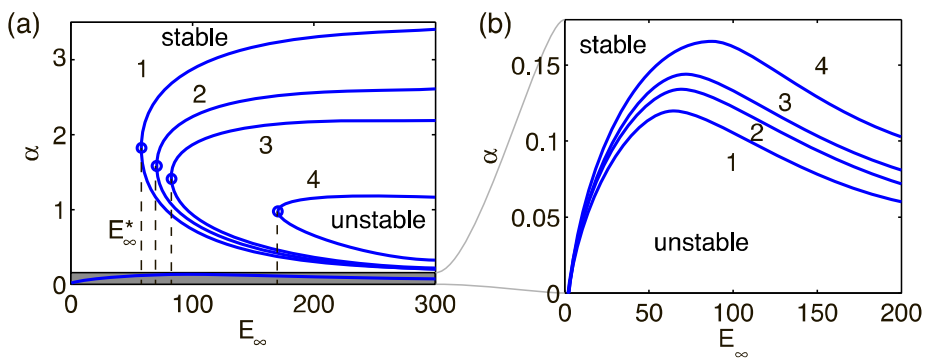

Figure 3. Marginal stability curves (a) short-wave region and (b) long-wave region for different values $\mu$ 1: $\mu=0$ $2: \mu=0.5,3: \mu=1,4: \mu=3.5$

Furthermore, the application of pressure gradient results in the destabilization of the onedimensional flow when the pressure driving force is oppositely directed to the electroosmotic flow. If the pressure driving force is codirected with the electroosmotic flow, then the one-dimensional solution is stabilized. This result is in good agreement with the experiment [2] (see Fig. 4).

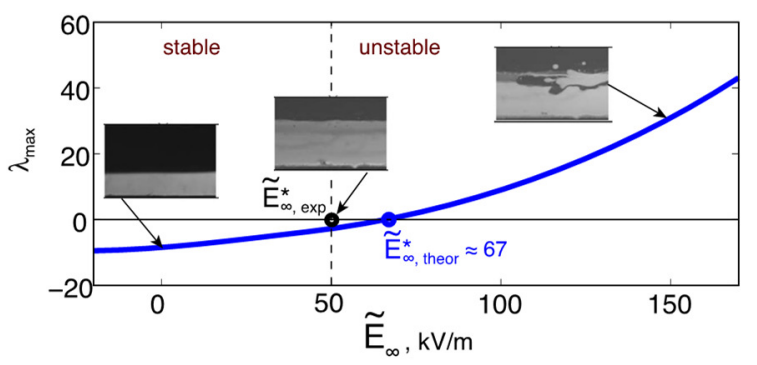

Figure 4. Comparison with experiments [2]. Maximal growth rate of the most dangerous perturbations $\lambda_{\max }$ vs dimensional external electric field $\tilde{E}_{\infty}$.

\section{Conclusion}

The stability of two-phase dielectric/electrolyte system, consisting of two immiscible liquids in microchannel, has been investigated. One-dimensional steady state has been founded. The numerical analysis provides the coexistance of long and short-wave instabilities. For the extreme cases there is a good correspondence of theoretical results in [1] and [5]. The first extreme case is the consideration of air as a dielectric liquid, and the second extreme case allows using of the Gouy-Chapman approximation. The theoretical values of critical external electric field are in good correspondence with the experimental data [2].

This work was supported, in part, by the Russian Foundation for Basic Research (Projects No. 1508-02483-a, No. 14-08-01171-a and 16-08-00643-a)

\section{References}

1. G.S. Ganchenko, E.A. Demekhin, M. Mayur, S. Amiroudine, Phys. Fluids 27, 062002 (2015)

2. H. Li, T.N. Wong, N.-T. Nguyen, Micro and Nanosystems 4, 14-24 (2012)

3. E.A. Demekhin, V.S. Shelistov, S.V. Polyanskikh, Phys. Rev. E 84, 036318 (2011)

4. V.S. Shelistov, N.V. Nikitin, G.S. Ganchenko, E.A. Demekhin, Dokl. Phys. 56, 538 (2011).

5. A. Navarkar, S. Amiroudine, M. Mayur, E. A. Demekhin, Microfluid Nanofluid 19, 813 (2015) 